\title{
Characterization and evolution of intestine injury at the anhepatic phase in portal hypertensive rats
}

\author{
GUIJUN REN ${ }^{1}$, XIAOYE YUAN ${ }^{2}$, XIN ZHAO ${ }^{1}$, QINGCHUN HAO ${ }^{1}$, JINGLIN CAO ${ }^{1}$, \\ YANG WANG $^{1}$, QINGJUN GAO ${ }^{1}$, JIAN DOU ${ }^{1}$ and QIANG ZENG ${ }^{1}$ \\ ${ }^{1}$ Department of Hepatobiliary Surgery, Third Affiliated Hospital of Hebei Medical University; \\ ${ }^{2}$ Department of Gerontology, Hebei General Hospital, Shijiazhuang, Hebei 050051, P.R. China
}

Received October 18, 2017; Accepted February 2, 2018

DOI: $10.3892 /$ etm.2018.6800

\begin{abstract}
The aim of the present study was to investigate the characteristics and progression of intestinal injury at the anhepatic phase in portal hypertensive rats. A total of 120 healthy male Wistar rats were purchased, with 15 rats in the normal control group and 105 rats were assigned to establish a prehepatic portal hypertension model. The 105 model rats were further divided into seven treatment groups following ischemia-reperfusion. Meanwhile, portal vein pressure, the area of lower esophageal mucosal vein, endotoxin levels in portal vein blood and the level of malondialdehyde (MDA) and superoxide dismutase (SOD) were measured. Morphology changes of the intestine were observed using optical microscopy and transmission electron microscopy. A portal hypertension rat model was successfully established. Furthermore, endotoxin, MDA and SOD level reached a peak at $12-24 \mathrm{~h}$ following reperfusion and then decreased gradually to normal levels at 1 week following reperfusion. However, cytological damage did not recover to preoperative level within 1 week. These findings suggest that intestinal injury was most severe within 12-24 $\mathrm{h}$ following ischemia-reperfusion and most indicators recovered to almost normal levels. Therefore, further study on the intestinal mucosal damage is required, with the aim to reduce the production of intestinal endotoxin.
\end{abstract}

\section{Introduction}

For over 30 years liver transplantation has been accepted as an effective treatment procedure for various end-stage liver diseases, such as hepatitis, cirrhosis and liver cancer (1-3). The 1- and 5-year survival rates following liver transplantation are 90 and $75 \%$, respectively (4). One challenge in performing

Correspondence to: Dr Qiang Zeng, Department of Hepatobiliary Surgery, Third Affiliated Hospital of Hebei Medical University, 139 Zi Qiang Road, Qiaoxi, Shijiazhuang, Hebei 050051, P.R. China E-mail: zengqiang4578@163.com

Key words: ischemia-reperfusion, intestine injury, portal hypertension rat model, anhepatic phase, liver transplantation liver transplantation is the potential for multiple organ injury, including acute renal failure, biliary injury and intestinal injury. Enteral and parenteral nutrition support have been widely recommended as an essential treatment to promote postoperative recovery following liver transplantation $(5,6)$. During liver transplantation, patients inevitably experience a short anhepatic phase, which may lead to ischemia/reperfusion (I/R) injury $(7,8)$. Interruption of the blood supply may induce tissue damage; however, restoration of blood flow does not relieve tissue damage, but leads to additional injury (9). The epithelial cells of the intestinal mucosa are particularly sensitive to hypoxia-ischemia upon intestinal congestion and the intestine is perhaps the most sensitive organ to I/R injury (10). A key consequence of intestinal IR injury is the breakdown of the intestinal barrier function, which results in bacterial translocation, endotoxemia, and uncontrolled release of inflammatory mediators and cytokines $(11,12)$. Diffusion of bacteria and toxins is a primary cause of systemic inflammatory response syndrome, multiple organ dysfunction syndrome and multiple organ failure (12).

Intestinal injury following liver transplantation may be caused by many risk factors, such as ischemia and hypoxia, endotoxemia, immune barrier damage, severe infection, hypercoagulability and I/R injury (13-15). I/R injury has been identified as the most notable reason, which also results in lung and kidney injury $(16,17)$. Recently, researchers have focused on clinical intervention and mechanism of intestinal injury, whereas histopathology analysis in intestinal injury has received less attention (18).

In the present study, a portal hypertension rat model was established to examine the hemodynamic changes and analyze the changes of structure and function with parameters associated with intestinal injury. The aim of the present study was to determine the time variation of intestinal injury in portal hypertensive rats that tolerate the anhepatic phase, provide rationale for the further study of intestinal injury and make improvements for clinical treatments following liver transplantation.

\section{Materials and methods}

Animals. A total of 120 healthy male Wistar rats (age, 8 weeks; weight, 250-300 g) were obtained from the Animal Center of 
Hebei Medical University (Shijiazhuang, China). Rats were housed at room temperature $\left(25 \pm 2^{\circ} \mathrm{C}\right)$, a relative humidity of $50 \pm 10 \%$, a 12-h light/dark cycle and free access to food and water. All animal protocols were performed with adherence to the guidelines provided by the Animal Ethics Requirements of Hebei Medical University (Shijiazhuang, China). The protocol was approved by the Ethics Committee of Third Affiliated Hospital of Hebei Medical University (Shijiazhuang, China).

Establishment of portal hypertension rat model. Portal hypertension rat models were established as previously described (17,19), with modifications. The 20-gauge blunt needle, self-made label tube and portal vein were ligated together, and then the needle was drawn to make the diameter of portal vein equal to the diameter of 20-gauge needle. Briefly, 120 healthy male Wistar rats were randomly divided into 8 groups: Normal control group (NCG), portal hypertension control group (PHCG), I/R $0 \mathrm{~h}$ (I/R0hG), $12 \mathrm{~h}$ (I/R12hG), 24 h (I/R24hG), 48 h (I/R48hG), 72 h (I/R72hG) and 1 week (I/R1wG) groups, with 15 rats in each group. The initial surgery was performed in 105 rats to establish the portal hypertension rat model, and the remaining 15 rats were assigned to the NCG group. The portal hypertension rat model was successfully established 3 weeks following the first surgery. Then, 15 model rats were randomly selected as the PHCG group, and the second surgery was performed on the other 90 model rats. Rats in the NCG or PHCG groups were sacrificed following establishment of the portal hypertension rat model. The 90 model rats were sacrificed at $0,12,24,48$ and $72 \mathrm{~h}$, and 1 week following removal of the occlusion and relevant indicators were detected.

Measurement of portal vein pressure and area of lower esophageal mucosal vein. Following a period of 3 weeks, 5 rats each from the NCG and PHCG groups were randomly selected and anesthetized via intraperitoneal injection of 2\% ketamine (200 mg/kg; Jiangsu Hengrui Medicine Co. Ltd., Lianyungang, China). Subsequently, a 2-cm rectus abdominis incision was made and the colon vein was located. A 3F epidural catheter was inserted into the trunk of the portal vein and portal vein pressure was measured with an eight-channel polygraph (RM-6000; Nihon Kohden, Tokyo, Japan). Lower esophageal tissues of NCG rats and PHCG rats were respectively fixed in $10 \%$ formalin solution for $24 \mathrm{~h}$ at room temperature and prepared into $5-\mu \mathrm{m}$ paraffin sections. Following $1 \mathrm{~h}$ hematoxylin and eosin $(\mathrm{H} \& \mathrm{E})$ staining at room temperature, the sections were observed under light microscopy at x100 magnification (BX51; Olympus Corporation, Tokyo, Japan) and the mean area of the submucosal vein per $\mathrm{mm}^{2}$ of the esophagus was calculated using CMIAS-2001B multifunctional color pathological image analysis system (version 4.0; Beihang University, Beijing, China).

Determination of endotoxin in portal vein blood. The endotoxin determination was performed in 15 rats from each group. Rats in NCG or PHCG groups were sacrificed following establishment of portal hypertension rat model. The other 90 model rats were sacrificed at $0,12,24,48$ and $72 \mathrm{~h}$, and 1 week following removal of the occlusion. The abdominal cavity was opened under asepsis, and $2 \mathrm{ml}$ blood sample was collected from the portal vein system. The blood samples were placed in sterile pyrogen-free test tubes and stored at $-20^{\circ} \mathrm{C}$. The bacterial endotoxin level was quantitatively analyzed using a limulus lysate test kit (Nanjing Jiancheng Bioengineering Institute, Nanjing, China), according to the manufacturer's protocol.

Measurement of malondialdehyde (MDA) and superoxide dismutase (SOD) level. The MDA and SOD measurement was performed in 15 rats from each group. Following collecting the small intestine of rats, $1 \mathrm{~g}$ intestinal tissues were weighed and homogenized with a glass homogenizer. The absorbance was determined using a UV-2550 spectrophotometer (Shimadzu Corporation, Kyoto, Japan). MDA was measured at $532 \mathrm{~nm}$ and SOD was measured at $560 \mathrm{~nm}$. All the above operations were performed at $0-4^{\circ} \mathrm{C}$.

Optical and electron microscopy examination on intestinal tissue. A total of 10 rats from each group were randomly selected and small intestine tissues were harvested following sacrifice. Tissues were fixed in $10 \%$ formalin solution for $24 \mathrm{~h}$ at room temperature, paraffin embedded, sliced (4- $\mu \mathrm{m}), \mathrm{H} \& \mathrm{E}$ stained at room temperature for $1 \mathrm{~h}$, and observed under light microscopy. In addition, 10 rats were randomly taken from each group and the small intestine tissues were cut into $1-\mathrm{mm}^{3}$ tissue blocks. These samples were subsequently fixed in $4 \%$ glutaraldehyde and $1 \%$ osmic acid solution for $4 \mathrm{~h}$ at $4{ }^{\circ} \mathrm{C}$, and dehydrated by acetone. Then samples were embedded in epoxy resin (Epon812), maintained at $37^{\circ} \mathrm{C}$ overnight and then $60^{\circ} \mathrm{C}$ for $24 \mathrm{~h}$ to produce ultra-thin sections $(60 \mathrm{~nm})$. Sections were stained at room temperature with uranyl acetate $(5 \%)$ for $30 \mathrm{~min}$ and then lead citrate stained for $10 \mathrm{~min}$. A Hitachi H-7500 transmission electron microscope at x20,000 magnification (Hitachi, Ltd., Tokyo, Japan) was used for ultrastructure examination.

Statistical analysis. Data were analyzed using SPSS 11.0 (SPSS, Inc., Chicago, IL, USA) and are presented as the mean \pm standard deviation. Comparisons were performed using one-way analysis of variance with Tukey's test or Student's t-test, as appropriate. $\mathrm{P}<0.05$ was considered to indicate a statistically significant difference.

\section{Results}

Measurement of portal vein pressure and area of lower esophageal mucosal vein. As presented in Fig. 1A, the portal pressure of $\mathrm{NCG}$ rats was $7.7 \pm 1.7 \mathrm{mmHg}$ and was significantly increased in PHCG rat model $(15.6 \pm 3.1 \mathrm{mmHg}$; $\mathrm{P}<0.05)$. Following removal of the catheter, portal vein pressure in $\mathrm{PHCG}$ group was $13.4 \pm 2.3 \mathrm{mmHg}$, which remained significantly higher than the NCG group $(\mathrm{P}<0.05)$. The mean area of esophageal submucosa vein in the NCG group was $5.6200 \pm 1.3010 \mu \mathrm{m}^{2}$ and the average area of the PHCG group was significantly higher, at $33.5820 \pm 6.4250 \mu \mathrm{m}^{2} \quad(\mathrm{P}<0.05$; Fig. 1B).

Analysis of endotoxin in portal vein blood. In order to analyze serum biochemical changes, endotoxin level of the eight groups were measured. Compared with the NCG or PHCG group, 

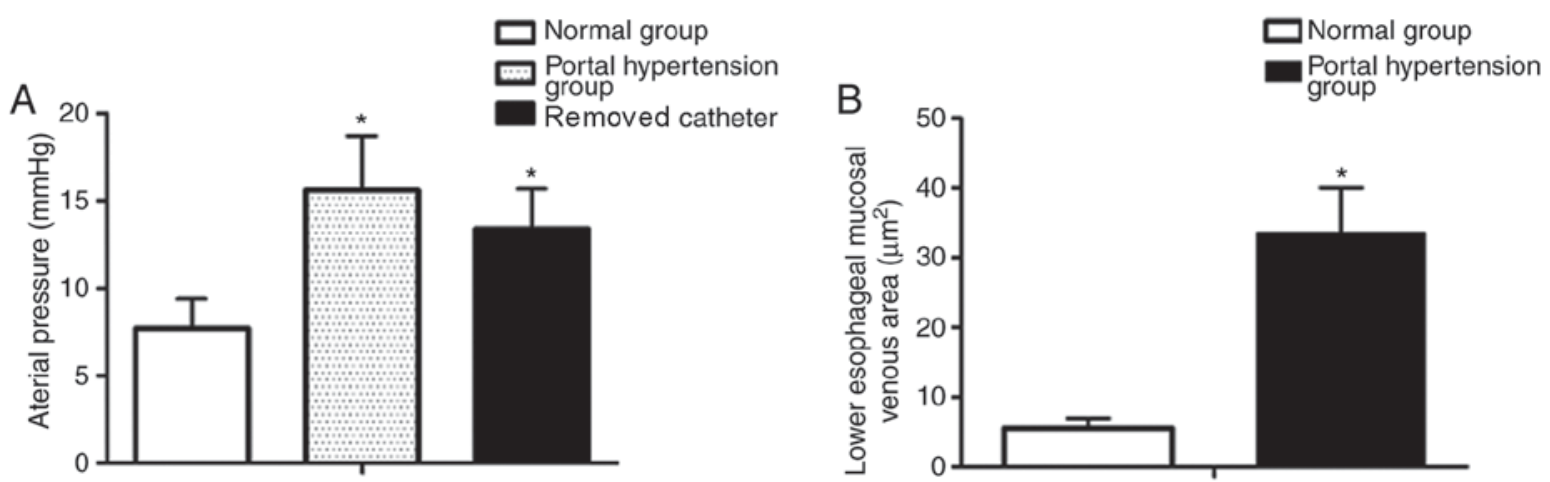

Figure 1. Measurement of (A) arterial blood pressure and (B) area of lower esophageal mucosal vein. Arterial blood pressure was monitored using BIOPAC systems. Data are presented as the mean \pm standard deviation of three replicate experiments. ${ }^{*} \mathrm{P}<0.05$ vs. normal group.

endotoxin in I/R12hG was significantly increased $(\mathrm{P}<0.05)$, and exhibited the highest level of all groups (Fig. 2). Furthermore, endotoxin revealed an increasing profile and reached a peak at $12 \mathrm{~h}$ following ischemia-reperfusion $(0.1202 \pm 0.0218 \mathrm{Eu} / \mathrm{ml})$. Subsequently, endotoxin decreased gradually to normal levels in I/R1wG. Furthermore, there were no significant differences among NCG, PHCG, and I/R1wG.

Analysis of MDA and SOD level. In order to further evaluate intestinal injury at the anhepatic phase, MDA and SOD levels were measured. Notably, the MDA of the intestine revealed a contrary profile to that of SOD (Fig. 3A and B). The SOD level of the intestine was lowest at I/R24hG and gradually increased over time following I/R. As expected, the SOD level of intestine in PHCG rats was decreased compared with NCG rats. The SOD level at 12 and $24 \mathrm{~h}$ was significantly decreased $(\mathrm{P}<0.05)$, compared with the NCG or PHCG groups, whereas the SOD level of intestine at 1 week was similar to the level of that in the PHCG group. There were no significant differences between the R1 w and PHCG groups.

Histopathological analysis of intestinal tissues. Paraffin-embedded sections of intestine tissues were observed using an optical microscope. As presented in Fig. 4, there was no marked abnormality in the structure of the ileal villus in NCG and PHCG. However, in I/R24hG, ileal mucosal villi were sparse and dislocated, and the villus height was significantly decreased, which was markedly different than the other groups. Furthermore, the lesions in I/R48hG and I/R72hG indicated that gradual recovery had occurred; however, $\mathrm{I} / \mathrm{R} 1 \mathrm{wG}$ did not recover to the preoperative level.

Cell ultrastructure in intestinal tissues. Electron microscopy observation demonstrated that there were no marked pathological changes between the ileal mucosa of NCG and PHCG. However, in I/R24hG, marked pathological manifestations were observed, including: The microvilli of the ileal mucosa were sparse and disordered; the intracellular mitochondria were swollen; the majority of the cristae were disappeared; mitochondrial membrane was partially damaged and the rough endoplasmic reticulum was expanded, appearing pool-like. Furthermore, in I/R0hG and I/R12hG, minor lesions were observed compared with $\mathrm{I} / \mathrm{R} 24 \mathrm{hG}$, such that the microvilli were partially detached and arranged irregularly, and the cytoplasm

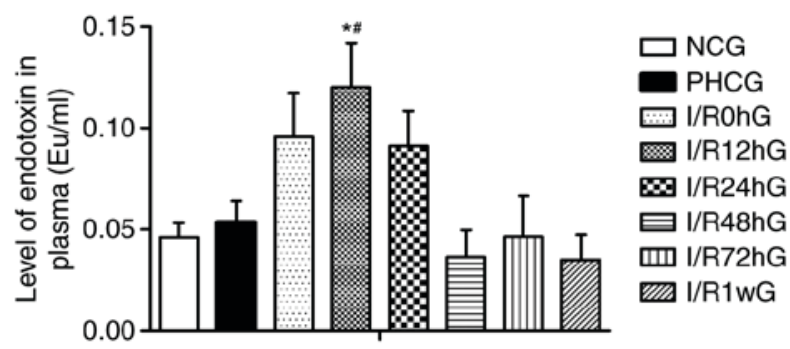

Figure 2. Analysis of endotoxin in portal vein blood. Data are presented as the mean + standard deviation of three replicate experiments. ${ }^{*} \mathrm{P}<0.05$ vs. NCG; ${ }^{\#} \mathrm{P}<0.05$ vs. PHCG. NCG, normal control group; PHCG, portal hypertension control group; I/R, ischemia reperfusion; I/R0hG, I/R $0 \mathrm{~h}$ group; I/R12hG, I/R 12 h group; I/R24hG, I/R 24 h group; I/R48hG, I/R 48 h group; I/R72hG, I/R 72 h group; I/R1wG, I/R 1 week group.

was edematous. Furthermore, in I/R48hG and I/R72hG, the lesions were gradually recovered. However, in $\mathrm{I} / \mathrm{R} 1 \mathrm{wG}$, the lesion has not recovered to pre-operative levels (Fig. 5).

\section{Discussion}

The intestinal mucosal barrier serves an important role in evaluating the illness state and the prognosis (20). At present, liver transplantation has been accepted as an established therapy for end-stage liver diseases (1-2). However, during the procedure, congestion for $\geq 45$ min may occur in the portal vein system and the gastrointestinal tract, which may lead to congestion, ischemia and reperfusion injury in small intestine. The I/R injury may lead to the release of oxygen-derived free radicals, endogenous infections, lipid overoxidation and calcium overload, which result in more serious intestinal mucosa injury and increasing postoperative mortality rate (2-4). A previous study reported that the intestine was probably the most sensitive organ to I/R injury (7). Therefore, the influence of intestine congestion and low perfusion pressure on the intestinal function of patients cannot be ignored; it is important to elucidate the pathological changes and recovery experience of intestinal injury in the anhepatic phase.

The use of a portal hypertension rat model can maximally simulate hemodynamic changes following liver transplantation. In the present study, the application of anterior hepatic portal hypertension model for anhepatic phase was primarily based on the following considerations: i) The portal hypertension rat 
A

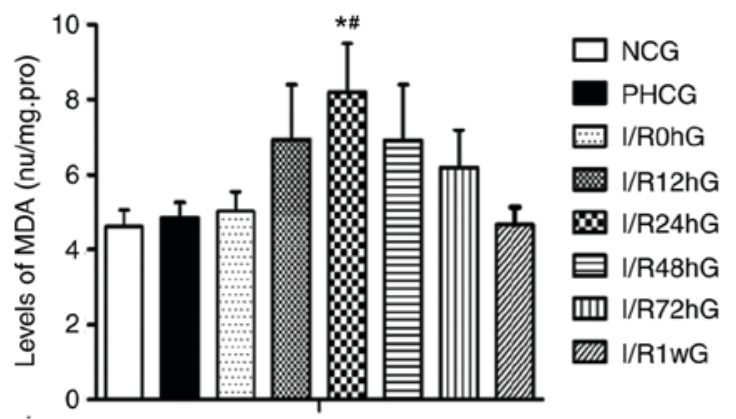

B

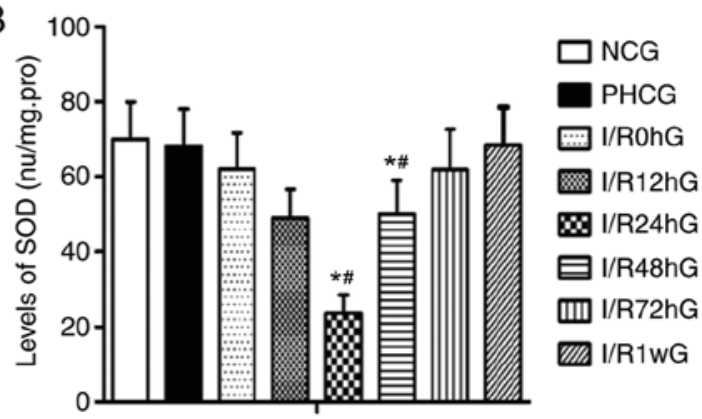

Figure 3. Analysis of (A) MDA and (B) SOD level. Data are presented as the mean + standard deviation of three replicate experiments. * $<<0.05$ vs. NCG; ${ }^{\#} \mathrm{P}<0.05$ vs. PHCG. MDA, malondialdehyde; SOD, superoxide dismutase; NCG, normal control group; PHCG, portal hypertension control group; I/R, ischemia reperfusion; I/R0hG, I/R 0 h group; I/R12hG, I/R 12 h group; I/R24hG, I/R 24 h group; I/R48hG, I/R 48 h group; I/R72hG, I/R 72 h group; I/R1wG, I/R 1 week group.
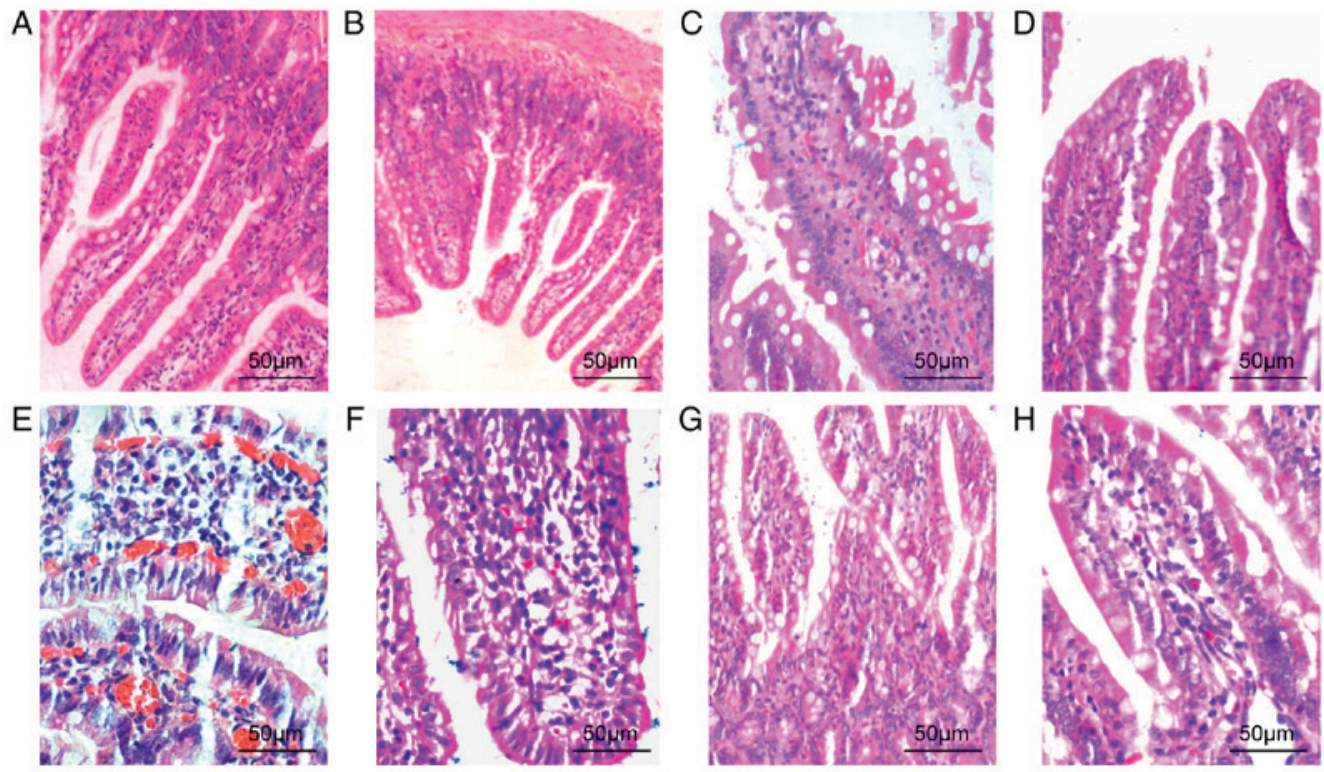

Figure 4. Histopathological analysis of intestine tissues with hematoxylin-eosin staining. (A) Normal control group; magnification, x200; (B) portal hypertensive control group; magnification, x100; (C) I/R 0 h group; magnification, x400; (D) I/R 12 h group; magnification, x200; (E) I/R $24 \mathrm{~h}$ group; magnification, x400; (F) I/R 48 h group; magnification, x400; (G) I/R 72 h group; magnification, x400; (H) I/R 1 week group; magnification, x400. I/R, ischemia reperfusion.

model has enhanced tolerance for surgery and is similar to that of human end-stage liver disease; ii) the model ensures high pressure of the portal vein system, and the normal structure and function of liver; iii) there are numerous factors, including intestinal congestion and low perfusion pressure, that affect the function of the small intestine following liver transplantation. The use of this model is suitable to evaluate the influence of anhepatic hypotension and inferior vena cava congestion on small intestine with portal vein shunt. In the previous experiments with the model, it may be difficult to locate the portal vein, which persisted following cutting the narrow line $(17,19)$. After constructing the model of portal hypertension secondary to liver cirrhosis, the portal vein could not be completely restored. However, the portal venous resistance will recover after clinical liver transplantation. To rectify this issue, the surgical procedure based on previous procedures $(17,19)$ was modified. The 20-gauge blunt needle, self-made label tube and portal vein were ligated together, and then the needle was drawn to make the diameter of portal vein equal to the diameter of the 20-gauge needle. In the second operation, the portal vein was easily located with label tube and subsequently removed, which avoided damage to the portal vein. The portal vein pressure measurement, angiography and esophageal submucosal vein image analysis demonstrated that the experimental portal hypertension model was successfully established.

The hemodynamic changes in rats following anhepatic phase were notable. In theory, blocking the inferior vena cava and portal vein will reduce mean arterial pressure and small intestine perfusion pressure. Following opening the bloodstream, the small intestine will be subjected to reperfusion injury. In the present study, the mean arterial pressure decreased to $56.3 \pm 2.8 \mathrm{mmHg}$ at the beginning of the anhepatic phase and the small intestine experienced a hypotension period during surgery. Marked pathological changes in the small intestine were observed at I/R24hG. For example, the mucosal villi were sparse, dislocated and disordered, which 

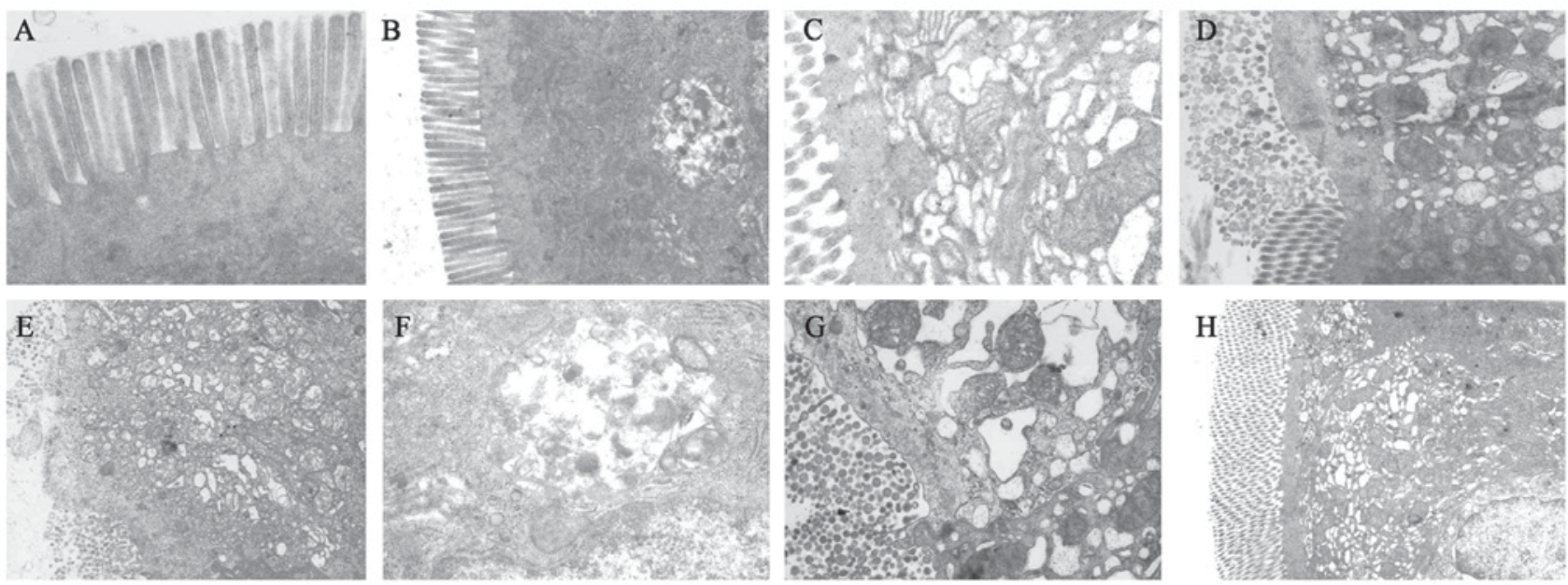

Figure 5. Cell ultrastructure analysis of intestine tissues. The ultrastructure of intestinal mucosal cells was observed by transmission electron microscopy. (A) Normal control group; original magnification, x20,000; (B) portal hypertensive control group; original magnification, x10,000; (C) I/R 0 h group; original magnification, x20,000; (D) I/R 12 h group; original magnification, x10,000; (E) I/R 24 h group; original magnification, x6,000; (F) I/R 48 h group; original magnification, x20,000; (G) I/R 72 h group; original magnification, x10,000; (H) I/R 1 week group; original magnification, x5,000. I/R, ischemia reperfusion.

was in line with electron microscopy analysis. However, plasma endotoxin level gradually returned to normal over $48 \mathrm{~h}$ and small intestine pathological changes significantly improved after 1 week, which suggested that small intestine dysfunction may recover in a short period. The recovery of small intestine function benefited from extensive collateral circulation. For example, the portal vein is able to communicate with the superior vena cava through fundus esophageal vein and azygos vein, and with the inferior vena cava through the gastric fundus esophageal vein $(21,22)$. Meanwhile, hemodynamic characteristics of portal hypertension demonstrated a high output and low resistance state, which allows it to tolerate a $50 \%$ decrease in cardiac output during the anhepatic phase (23). In the present study, following 1 week of reperfusion, the serology had returned to normal, but the histology of the small intestine was not restored to the normal state. This suggested that the intestinal function support should be enhanced within 1 week after the non-bypass liver transplantation.

The gastrointestinal tract is one of the target organs of $\mathrm{I} / \mathrm{R}$ injury and is often described as the central organ of surgical stress and the initiating organ of multiple organ failure (24). The intestinal mucosa is rich in blood supply to ensure the absorption of nutrients and the maintenance of normal barrier function (25). Due to certain characteristics of intestinal villus oxygen exchange, including that the oxygen tension at the tip of villus is lower than that of the artery, cells have a high sensitivity to hypoxia and ischemia (26). During the anhepatic phase of conventional non-bypass liver transplantation, the blood pressure decreases. In order to ensure sufficient blood supply of vital organs, the effective circulation volume of intestinal mucosa and submucosa decreases, which results in impaired intestinal mucosal barrier function (27). Electron microscopy results demonstrated that the mitochondria were markedly diseased, which lead to an increase of anaerobic metabolism and the acidosis of the mucous membrane. This indicated that the disorder of oxygen metabolism induced the energy metabolism of intestinal mucosal cells. The decrease of ATP results in the destruction of the cytoskeleton and the increase of intracellular calcium concentration, which further results in increased cell permeability (28). These results suggest that mitochondrial damage induced by ischemia and hypoxia may be one of the most important mechanisms of small intestinal injury in rats with portal hypertension undergoing the anhepatic phase.

Following removal of the inferior vena cava and hepatic portal occlusion, the microvilli of the small intestinal mucosa were extensively detached, resulting in disruption of the cell barrier and mucus barrier. Disruption of the mucus barrier leads to a decrease in the function of the small intestinal mucosa against proteases, thereby exacerbating the damage to the intestinal mucosa (29). It has been reported that the mass release of oxygen free radicals in local tissues is a major cause of intestinal damage (30). MDA is the degradation product of lipid peroxide, and its presence marks the process of lipid peroxidation in the organism (31). SOD is an antioxidant enzyme that produces hydrogen peroxide $\left(\mathrm{H}_{2} \mathrm{O}_{2}\right)$, and its concentration can be reduced when it is used to scavenge superoxide anion to produce $\mathrm{H}_{2} \mathrm{O}_{2}$ (32). In Li et al's previous study (33), the concentration of MDA in each group gradually increased following reperfusion, which is typically considered the result of a marked release of free radicals and the accumulation of lipid peroxide products following pan-intestinal I/R. The present study demonstrated that the content of MDA initially increased prior to decreasing in portal hypertensive rats following the anhepatic phase, which is consistent with Li's study. However, the content of SOD initially decreased first and subsequently increased, which indicated that oxygen free radicals were associated with the damage of the intestinal mucosal barrier. Impaired intestinal barrier function may result in endotoxemia (9). In the present study, at $1 \mathrm{~h}$ following blocking the inferior vena cava and hepatic portal vein, the endotoxin in the portal vein was markedly increased, which indicated that the permeability of intestinal mucosa was increased and the intestinal mucosal barrier was damaged. Endotoxin, a lipopolysaccharide located in the cell walls of gram-negative bacteria, has a wide range of biological activity 
that included aggravating mucosal function damage by activating vasoactive substances and adenosine cyclase on the membranes of small intestinal epithelial cells (34). Endotoxins that are over-produced during liver transplantation bind to the primary receptor, toll-like receptor 4 , which is important in post-liver transplantation intestinal injury. Yuan et al (35) recently demonstrated that levels of enterogenous endotoxin peaked at $8 \mathrm{~h}$ following reperfusion, the point at which the intestine exhibited the most notable damage. However, in the present study, the levels of endotoxin reached their peak at $12 \mathrm{~h}$ following reperfusion. The difference between the two studies may result from the different rat models used. Intestinal endotoxemia, which is caused by intestinal mucosal barrier damage, has been gradually recognized and it serves an important role in the pathogenesis of multiple organ failure $(36,37)$. Therefore, further study on the intestinal mucosal damage, aiming to reduce the production of intestinal endotoxin is crucial to clinical research.

According to its pathogenesis, it is possible to alleviate the injury of the small intestine by applying drugs to prevent the I/R injury of intestinal tissue mediated by oxygen free radicals, protect the intestinal mucosal barrier and strengthen the anti-infection treatment. In the present study, the effect of the anhepatic phase on the function of the small intestine was observed only for $1 \mathrm{~h}$. However, the safe duration of the anhepatic phase of the liver requires further study.

In conclusion, the present study demonstrated that small intestine injury of portal hypertensive rats with anhepatic phase was most severe within $12-24 \mathrm{~h}$ following I/R and the small intestine injury could be ameliorated. The present findings suggest that hemodynamic change is one of the important reasons for intestinal injury; however the mechanism of this intestinal injury must be elucidated in future clinical research studies.

\section{Acknowledgements}

Not applicable.

\section{Funding}

No funding was received.

\section{Availability of data and materials}

The datasets used and/or analyzed during the current study are available from the corresponding author on reasonable request.

\section{Authors' contributions}

GR and QZ conceived the study design. GR, XY and QZ wrote the manuscript. QH, GR and XZ interpreted the data. GR, QZ analyzed the data. JC, YW, QG and JD collected the data. All authors have read and approved the manuscript.

\section{Ethics approval and consent to participate}

The present study was approved by the Ethics Committee of the Third Affiliated Hospital of Hebei Medical University (Shijiazhuang, China).

\section{Patient consent for publication}

Not applicable.

\section{Competing interests}

The authors declare that they had no competing interests.

\section{References}

1. Chi X, Yao W, Xia H, Jin Y, Li X, Cai J and Hei Z: Elevation of $\mathrm{HO}-1$ expression mitigates intestinal ischemia-reperfusion injury and restores tight junction function in a rat liver transplantation model. Oxidative Med Cell Longevity 2015: Article ID 986075 , 2015.

2. Åberg F, Isoniemi $\mathrm{H}$ and Höckerstedt K: Long-term results of liver transplantation. Scand J Surg 100: 14-21, 2011.

3. Mazzaferro V, Regalia E, Doci R, Andreola S, Pulvirenti A, Bozzetti F, Montalto F, Ammatuna M, Morabito A and Gennari L: Liver transplantation for the treatment of small hepatocellular carcinomas in patients with cirrhosis. N Engl J Med 334: 693-700, 1996.

4. Wiesner RH and Menon KV: Late hepatic allograft dysfunction. Liver Transpl 7 (11 Suppl 1): S60-S73, 2001.

5. Hasse JM, Blue LS, Liepa GU, Goldstein RM, Jennings LW, Mor E, Husberg BS, Levy MF, Gonwa TA and Klintmalm GB: Early enteral nutrition support in patients undergoing liver transplantation. JPEN J Parenter Enteral Nutr 19: 437-443, 1995.

6. Reilly J, Mehta R, Teperman L, Cemaj S, Tzakis A, Yanaga K, Ritter P, Rezak A and Makowka L: Nutritional support after liver transplantation: A randomized prospective study. Jpen J Parenter Enteral Nutr 14: 386-391, 1990

7. Glanemann M, Langrehr J, Kaisers U, Schenk R, Müller A, Stange B, Neumann U, Bechstein WO, Falke K and Neuhaus P: Postoperative tracheal extubation after orthotopic liver transplantation. Acta Anaesthesiol Scand 45: 333-339, 2001.

8. Ren Z, Cui G, Lu H, Chen X, Jiang J, Liu H, He Y, Ding S, Hu Z, Wang $W$ and Zheng S: Liver ischemic preconditioning (IPC) improves intestinal microbiota following liver transplantation in rats through 16s rDNA-based analysis of microbial structure shift. PLoS One 8: e75950, 2013.

9. Kageyama S, Hata K, Tanaka H, Hirao H, Kubota T, Okamura Y, Iwaisako K, Takada Y and Uemoto S: Intestinal ischemic preconditioning ameliorates hepatic ischemia/reperfusion injury in rats: Role of heme oxygenase 1 in the second window of protection. Liver Transpl 21: 112-122, 2015.

10. Stringa P, Lausada N, Romanin D, Machuca M, Cabanne A, Rumbo $\mathrm{M}$ and Gondolesi G: Defining the nonreturn time for intestinal ischemia reperfusion injury in mice. Transplant Proc 44: 1214-1217, 2012.

11. Shi H, Li R, Qiang J, Li Y, Wang L and Sun R: Computed tomography perfusion imaging detection of microcirculatory dysfunction in small intestinal ischemia-reperfusion injury in a porcine model. PLoS One 11: e0160102, 2016.

12. Cerqueira NF, Hussni CA and Yoshida WB: Pathophysiology of mesenteric ischemia/reperfusion: A review. Acta Cir Bras 20: 336-343, 2005.

13. Xie Y, Chen H, Zhu B, Qin N, Chen Y, Li Z, Deng M, Jiang H, $\mathrm{Xu} \mathrm{X}$, Yang J, et al: Effect of intestinal microbiota alteration on hepatic damage in rats with acute rejection after liver transplantation. Send to Acta Cir Bras 68: 871-880, 2014.

14. Yu MH, Yu XL, Chen CL, Gao LH, Mao WL, Yan D, Chen Y, Sheng JF, Li LJ and Zheng SS: The change of intestinal microecology in rats after orthotopic liver transplantation. Zhonghua Wai Ke Za Zhi 46: 1139-1142, 2008 (In Chinese).

15. Soubrane O, el Meteini M, Devictor D, Bernard O and Houssin D: Risk and prognostic factors of gut perforation after orthotopic liver transplantation for biliary atresia. Liver Transpl Surg 1: 2-9, 1995.

16. Markel TA, Crafts TD, Jensen AR, Hunsberger EB and Yoder MC: Human mesenchymal stromal cells decrease mortality after intestinal ischemia and reperfusion injury. J Surg Res 199: 56-66, 2015.

17. Zhao X, Zeng Q, Ren G, Cao J, Dou J and Gao Q: Pulmonary injury at the anhepatic phase without veno-venous bypass in portal hypertensive rats. Clin Exp Hypertens 38: 624-630, 2016. 
18. Wang YC, Jin QM, Kong WZ and Chen J: Protective effect of salvianolic acid B on NASH rat liver through restoring intestinal mucosal barrier function. Int J Clin Exp Pathol 8: 5203-5209, 2015.

19. Xin Z, Jian D and Gao QJ: Establishment of a reversible model of prehepatic portal hypertension in rats. Exp Ther Med 12: 939-944, 2016

20. Ammori BJ: Role of the gut in the course of severe acute pancreatitis. Pancreas 26: 122-129, 2003.

21. Robinson JW and Mirkovitch V: The recovery of function and microcirculation in small intestinal loops following ischaemia. Gut 13: 784-789, 1972.

22. Blikslager AT, Moeser AJ, Gookin JL, Jones SL and Odle J: Restoration of barrier function in injured intestinal mucosa. Physiol Rev 87: 545-564, 2007.

23. Li X, Benjamin IS and Alexander B: The relationship between intrahepatic portal systemic shunts and microsphere induced portal hypertension in the rat liver. Gut 42: 276-282, 1998.

24. Beath S, Pironi L, Gabe S, Horslen S, Sudan D, Mazeriegos G, Steiger E, Goulet $\mathrm{O}$ and Fryer J: Collaborative strategies to reduce mortality and morbidity in patients with chronic intestinal failure including those who are referred for small bowel transplantation. Transplantation 85: 1378-1384, 2008.

25. Holmberg FEO, Pedersen J, Jørgensen P, Soendergaard C Jensen $\mathrm{KB}$ and Nielsen $\mathrm{OH}$ : Intestinal barrier integrity and inflammatory bowel disease: Stem cell-based approaches to regenerate the barrier. J Tissue Eng Regen Med 12: 923-935, 2018.

26. Faingold R, Cassia G, Prempunpong C, Morneault L and Sant'Anna GM: Intestinal ultrasonography in infants with moderate or severe hypoxic-ischemic encephalopathy receiving hypothermia. Pediatr Radiol 46: 87-95, 2016.

27. Delva E, Barberousse JP, Nordlinger B, Ollivier JM, Vacher B, Guilmet $\mathrm{C}$ and Huguet C: Hemodynamic and biochemical monitoring during major liver resection with use of hepatic vascular exclusion. Surgery 95: 309-318, 1984.
28. Wu X, Ren J, Chen G, Wu L, Song X, Li G, Deng Y, Wang G, Gu G and Li J: Systemic blockade of P2X7 receptor protects against sepsis-induced intestinal barrier disruption. Sci Rep 7: 4364, 2017.

29. Shu X, Zhang J, Wang Q, Xu Z and Yu T: Glutamine decreases intestinal mucosal injury in a rat model of intestinal ischemia-reperfusion by downregulating HMGB1 and inflammatory cytokine expression. Exp Ther Med 12: 1367-1372, 2016.

30. Shen ZY, Zhang J, Song HL and Zheng WP: Bone-marrow mesenchymal stem cells reduce rat intestinal ischemia-reperfusion injury, ZO-1 downregulation and tight junction disruption via a TNF- $\alpha$-regulated mechanism. World J Gastroenterol 19: 3583-3595, 2013 .

31. Draper HH and Hadley M: Malondialdehyde determination as index of lipid peroxidation. Methods Enzymol 186: 421-431, 1990.

32. Jamdhade AR, Sunkar R and Hivrale VK: Zymographic method for distinguishing different classes of superoxide dismutases in plants. Methods Mol Biol 1631: 221-227, 2017.

33. Li CM,Zhang XH, Ma XJ and Luo M: Limb ischemic postconditioning protects myocardium from ischemia-reperfusion injury. Scand Cardiovasc J 40: 312-317, 2006.

34. Yeh YC, Yu LC, Wu CY, Cheng YJ, Lee CT, Sun WZ, Tsai JC and Lin TY; NTUH Center of Microcirculation Medical Research (NCMMR): Effects of endotoxin absorber hemoperfusion on microcirculation in septic pigs. J Surg Res 211: 242-250, 2017.

35. Yuan DD, Chi XJ, Jin Y, Li X, Ge M, Gao WL, Guan JQ, Zhang AL and Hei ZQ: Intestinal injury following liver transplantation was mediated by TLR4/NF- $\mathrm{BB}$ activation-induced cell apoptosis. Mol Med Rep 13: 1525-1532, 2016.

36. Lee HT, Kim M, Kim JY, Brown KM, Ham A, D'Agati VD and Mori-Akiyama Y: Critical role of interleukin-17A in murine intestinal ischemia-reperfusion injury. Am J Physiol Gastrointest Liver Physiol 304: G12-G25, 2013.

37. Feng AW, Gao W, Zhou GR, Yu R, Li N, Huang XL, Li QR and Li JS: Berberine ameliorates COX-2 expression in rat small intestinal mucosa partially through PPAR $\gamma$ pathway during acute endotoxemia. Int Immunopharmacol 12: 182-188, 2012. 